\title{
Groups with all Subgroups Subnormal or Nilpotent-by-Chernikov
}

\author{
HOWARD SMITH
}

It was proved by Asar in [1] that a locally nilpotent group in which every proper subgroup is nilpotent-by-Chernikov is itself nilpotent-by-Chernikov. It is easy to see that the main problem here lies with establishing solubility, since a non-trivial soluble group $G$ has a homomorphic image that is either cyclic or a Prüfer $p$-group, and if $M \triangleleft N \triangleleft G$, with $M$ nilpotent and both $G / N$ and $N / M$ Chernikov, then $M^{G}$ is easily shown to be nilpotent, and of course $G / M^{G}$ is Chernikov. In view of earlier results from [7], Asar's result completes the proof that a locally graded group with all proper subgroups nilpotent-by-Chernikov is nilpotent-by-Chernikov; in particular, a locally graded group with all proper subgroups nilpotent is soluble-by-finite.

Now, by a well-known result due to Möhres [6], a group $G$ with all subgroups subnormal is soluble, and in [9] and [10] the question was considered as to what might be said about a group $G$ in which every subgroup is either subnormal or nilpotent. With regard to establishing solubility, the main two results in [10] were that a locally soluble-byfinite group with all subgroups subnormal or nilpotent is itself soluble (Theorem 1), and a locally graded group in which every subgroup is either nilpotent or subnormal of defect at most $n$ is also soluble (Theorem 2). (Let us note here that there is an error, but one that is easily corrected, in the proof of Lemma 2.2 of [10], in that the appeal to Lemma 1.2 at the beginning of the second paragraph really requires an amended version of Lemma 1.2, where solubility replaces nilpotency throughout; the details are almost identical. A similar remark applies to the proof of the Proposition in [9], which invokes Lemma 3 of that paper, but here an alternative correction is to include in the statement of

Indirizzo dell'A.: Department of Mathematics, Bucknell University, Lewisburg, PA 17837, U.S.A.

E-mail: howsmith@bucknell.edu 
Lemma 4 of [9] the hypothesis that every soluble subgroup of $G$ be nilpotent.)

Further results in [9] and [10] are concerned with establishing rather more than solubility, and we do not concern ourselves in the present paper with attempting to generalize those results but postpone treatment to a couple of forthcoming articles. Our purpose here is to investigate whether a group with all subgroups either subnormal or nilpotent-by-Chernikov is soluble-by-finite, and as companion results to Theorems 1 and 2 of [10] we have the following.

THEOREM 1. Let $G$ be a locally soluble-by-finite group in which every subgroup is either subnormal or nilpotent-by-Chernikov. Then $G$ is soluble-by-finite and is either soluble or nilpotent-by-Chernikov.

THEOREM 2. Let G be a locally graded group in which every subgroup is either nilpotent-by-Chernikov or subnormal of defect at most $n$, where $n$ is some fixed positive integer. Then $G$ is soluble-by-finite and is either soluble or nilpotent-by-Chernikov.

As is the case for Theorem 1 of [10], Theorem 1 above generalizes quite easily to the case of groups $G$ that are locally generalized radical, that is, every finitely generated subgroup of $G$ is the union of an ascending series of subgroups whose factors are either locally nilpotent or locally finite. To prove this, it is enough, in view of Theorem 1 , to show that such a group $G$ is locally soluble-by-finite, and to this end we may assume that $G$ is generalized radical. By (possibly transfinite) induction on the length of an appropriate series of $G$, we may assume that there is a normal subgroup $K$ of $G$ with $G / K$ locally nilpotent or locally finite and $K$ locally soluble-byfinite and hence, by Theorem 1 , soluble-by-finite. Since $G / K$ is also soluble-by-finite it follows that $G$ is soluble-by-finite.

We recall from [10] that it is a most difficult problem to decide whether a locally graded group with all subgroups subnormal or nilpotent need be soluble, since it is not known whether there exists an infinite finitely generated residually finite $p$-group with all subgroups either finite (and hence nilpotent) or of finite index (and hence subnormal). This is our justification for introducing the bound on subnormal defects in the statement of Theorem 2. From now on we shall denote the class of nilpotent-byChernikov groups by $\mathfrak{i}$.

Our first result is easy to prove and is used several times during the course of our discussion. 
LEMMA 1. Let $G$ be a group in which every subgroup is either subnormal or in $\mathfrak{N C}$, and let $K$ be a non- $\mathfrak{N}$-subgroup of $G$. Then the dth term $G^{(d)}$ of the derived series of $G$ is contained in $K$, for some integer $d$, and if $G$ is not soluble-by-finite then every subgroup of $G / G^{(d)}$ is subnormal.

Proof. There is a finite subnormal series from $K$ to $G$, and each factor has all subgroups subnormal and is therefore soluble [6]; it follows that $G^{(d)} \leq K$ for some $d$. If $G$ is not soluble-by-finite then neither is $G^{(d)}$, so $G^{(d)} \notin \mathfrak{N C}$ and the result follows.

LEMMA 2. Let $G$ be a locally nilpotent group that is also locally finite, and suppose that every subgroup of $G$ is either subnormal or in $\mathfrak{N C}$ (respectively, subnormal or soluble). Then either $G$ is in $\mathfrak{N} 5$ (respectively, $G$ is soluble) or there is a primary component $G_{p}$ of $G$ such that $G_{p}$ is not in $\mathfrak{N C}$ (respectively, is not soluble) and $G=G_{p} \times K$, for some $\mathfrak{N}$-subgroup $K$.

Proof. Firstly we note that every $\mathfrak{N}$-subgroup of $G$ is soluble. Now suppose that every subgroup of $G$ is subnormal or in $\Re C^{5}$ and that some $G_{p}$ is not in $\mathfrak{N}$. Then every subgroup of $G / G_{p}$ is subnormal and so $G / G_{p} \in \mathfrak{N C}[3]$ and $G$ has the structure indicated. Suppose, on the other hand, that every primary component is in $\mathfrak{N} S$ but that $G$ is not. Either there are infinitely many $p$-components $G_{p_{i}}$ that are all nilpotent and are such that the class of $G_{p_{i}}$ increases with $i$, or there are infinitely many $p$-components $G_{p_{i}}$ that are not nilpotent. In either case we write $G=H_{1} \times H_{2}$, where each $H_{j}$ contains infinitely many of the $G_{p_{i}}$. It is clear that $H_{1}$ is not in $\mathfrak{N C}$ (since a Chernikov group involves just finitely many primes), but $H_{1} \cong G / H_{2}$, which has all subgroups subnormal and is therefore in $\mathfrak{N}$, again by [3]. This contradiction concludes the proof in the case where all subgroups are subnormal or in $\mathfrak{N C}$.

If every subgroup of $G$ is subnormal or soluble then much the same argument works: if $G_{p}$ is insoluble then $G / G_{p}$ has all subgroups subnormal and is therefore in $\mathfrak{N C}$ [3], and if $G_{p}$ is soluble for all primes $p$ but $G$ is not soluble then we may write $G=H_{1} \times H_{2}$ where this time each $H_{j}$ contains primary components of unbounded derived length. Again $H_{1} \cong G / H_{2}$, which is soluble [6], and a contradiction ensues.

Here is another result that is used on more than one occasion.

LEMMA 3. Let $G$ be a locally graded group in which every subgroup is either subnormal or in $\mathfrak{i C}$, let $R$ denote the soluble residual of $G$, and suppose that $G / R$ is soluble. Then $G$ is soluble-by-finite. 
Proof. If $G$ is not soluble-by-finite then neither is $R$, and so $R \notin \Re(5$. By results from [1] and [7] (as referred to at the beginning of our discussion), $R$ has a proper non- $\mathfrak{N}(-$-subgroup $S$, and since $S$ is subnormal in $R$ we have $S^{R}<R$ and every subgroup of $R / S^{R}$ subnormal, so $R / S^{R}$ is soluble, contradicting the fact that $R$ is perfect.

Our next result is a generalization of Lemma 1.2 of [10], referred to above. We have seen that Lemma 2.2 of [10] really required an adaptation of Lemma 1.2 (i.e. a "soluble version"), and we now state and prove a result that covers both this amended version and other cases. The following may be of use in further discussions of the kind presented here. Notice, for example, that the class $\mathfrak{N C}$ is an example of a class $\mathfrak{X}$ satisfying the given criterion. Notice also that if every subgroup of the group $G$ is subnormal but $G \notin \mathfrak{X}$ then the existence of such a triple $(K, F, r)$ as described in the proposition below follows from a result that is primarily due to Brookes [2] - see, for example, Lemma 3 of [3].

Proposition 1. Let $\mathfrak{X}=\bigcup_{i=1}^{\infty} \mathfrak{X}_{i}$ be a class of groups, where each class $\mathfrak{X}_{i}$ is $\langle s, L\rangle$-closed and $\mathfrak{X}_{i} \subseteq \mathfrak{X}_{i+1}$ for all $i$. Let $G$ be a group in which every subgroup is either subnormal or in $\mathfrak{X}$, and suppose that $G \notin \mathfrak{X}$. Then there is a positive integer $r$, a non- $\mathfrak{X}$ subgroup $K$ of $G$, and a finitely generated subgroup $F$ of $K$ such that, for every non-X subgroup $L$ of $K$ that contains $F, L$ is subnormal of defect at most $r$ in $K$.

Proof. Suppose the result false and set $K_{0}=G, H_{0}=1$. Assume that for some $i \geq 1$ we have subgroups $K_{i-1}, H_{i-1}$ of $G$, with $H_{i-1}$ finitely generated and contained in $K_{i-1}$, which is not in $\mathfrak{X}_{i}$. By hypothesis there is a non-X-Xubgroup $K_{i}$ of $K_{i-1}$ with $H_{i-1} \leq K_{i}$ and $K_{i}$ of defect greater than $i$ in $K_{i-1}$. Choose $x_{i} \in\left[K_{i-1, i} K_{i}\right] \backslash K_{i}$; then $x_{i} \in\left[K_{i-1, i} L_{i}\right]$ for some finitely generated subgroup $L_{i}$ of $K_{i}$, and (by $L$-closure) we may choose $L_{i}$ so that $L_{i} \notin \mathfrak{X}_{i}$. Set $H_{i}=\left\langle H_{i-1}, L_{i}\right\rangle$. Thus we have defined $K_{i}$ and $H_{i}$ inductively for all $i \geq 1$. Write $H=\bigcup_{i=1}^{\infty} H_{i}$; then $H \notin \mathfrak{X}$, and so $H$ is subnormal in $G$, of defect $d$, say. Now $x_{d} \in\left[K_{d-1, d} H_{d}\right] \leq\left[G,{ }_{d} H\right] \leq H$, and so $x_{d} \in H_{i}$ for some $i$, which we may choose to be greater than $d$. Thus $x_{d} \in H_{i} \leq K_{i} \leq K_{d}$, a contradiction. The result follows.

Proposition 2. Let $G$ be a locally nilpotent group in which every subgroup is either subnormal or nilpotent-by-Chernikov.Then $G$ is soluble. 
Proof. If $T$ denotes the torsion subgroup of $G$ then every subgroup of the torsion-free group $G / T$ is subnormal or nilpotent; it follows from Theorem 2 of [9] that $G / T$ is nilpotent. Thus we may assume that $G$ is periodic, and by Lemma 2 we may further suppose that $G$ is a $p$-group for some prime $p$. Assume the result false and let $R$ denote the soluble residual of $G$. By Lemma $3, G / R$ is insoluble, and we may factor and hence assume that $G$ is residually soluble.

By Proposition 1, with $\mathfrak{X}$ the class of soluble groups, we may also assume that there is a positive integer $r$ and a finite subgroup $F$ of $G$ such that every insoluble subgroup of $G$ that contains $F$ is subnormal of defect at most $r$ in $G$. Let $U$ be an arbitrary finite subgroup of $G$ containing $F$. We claim that $U$ is subnormal in $G$. First note that there is a normal subgroup $N$ of $G$ such that $G / N$ is soluble and $U \cap N=1$, and since $N$ is not soluble we have $U N$ subnormal in $G$. We may suppose that $G=U N$, so that $G / N$ is finite and hence nilpotent, say $\gamma_{k}(G) \leq N$. There is a chain $N>N_{1}>N_{2}>\ldots$ of normal subgroups of $G$ with each $G / N_{i}$ soluble and $\bigcap_{i=1}^{\infty} N_{i}=1$, and for each $i$ we have $F \leq U N_{i}$ and $U N_{i}$ insoluble, and so $\left[G_{r} U N_{i}\right] \leq U N_{i}$ for all $i$. Thus $[G, r+k] \leq \bigcap_{i=1}^{\infty} U N_{i} \cap N=\bigcap_{i=1}^{\infty} N_{i}(U \cap N)=1$, so $U$ is subnormal in $G$, as claimed. Since every finite subgroup of $G$ is contained in such a subgroup $U$, we see that $G$ is a Baer group.

Now, as in the proof of Lemma 5 of [5], if $G$ contains a hyperabelian subgroup $H$ that is not soluble then it contains an insoluble subgroup $H$ that has hyperabelian length $\omega$. By means of an application to $H$ of Proposition 1, if necessary, we may (by re-labelling) assume that $G=H$. But now $F$ is contained in some normal soluble subgroup $H_{0}$ of $G$, and if $H_{0}$ has derived length $n$ then we may choose $H_{0}$ maximal normal in $G$ with respect to having derived length $n$, in which case $G / H_{0}$ is also residually soluble. Let $V$ be an arbitrary normal subgroup of $G$ that contains $H_{0}$ and is such that $G / V$ is soluble. Then $V$ is not soluble and so every subgroup of $G / V$ is subnormal of defect at most $r$ in $G / V$. By Roseblade's Theorem [8], $G / V$ is nilpotent of bounded class, and so $G / H_{0}$ is nilpotent and we have the contradiction that $G$ is soluble. It follows that every hyperabelian subgroup of $G$ is soluble.

Again we argue as in [5]: let $x$ be an arbitrary element of $G$. Then $\langle x\rangle$ is subnormal and if $\langle x\rangle^{G}$ is not soluble then (by looking at the normal closure series of $\langle x\rangle$ in $G$ ) we see that there are subgroups $A, B, C$ of $G$, with $A$ soluble, $A \triangleleft B \triangleleft C, B=A^{C}$ and $B$ insoluble. Since $B$ is a product of normal subgroups, each of which is a conjugate of $A$, we have $B$ hyperabelian and 
hence soluble, and by this contradiction $\langle x\rangle^{G}$ is soluble. Since $x$ was arbitrary we now have $G$ hyperabelian and hence soluble, and this contradiction completes the proof of Proposition 2.

LeMma 4. Let $G$ be a group in which every subgroup is either subnormal or nilpotent-by-Chernikov. Then the product $V$ of all normal $\mathfrak{N}$ (Ssubgroups of $G$ is soluble-by-finite, and if $G$ is not soluble then $V$ is nilpotent-by-Chernikov.

PRoof. Let $V_{\lambda}$ be a normal $\mathfrak{N} \subseteq$-subgroup of $G$. Then there are characteristic subgroups $L_{\lambda}, D_{\lambda}$ of $V_{\lambda}$ such that $L_{\lambda} \leq D_{\lambda}, L_{\lambda}$ is locally nilpotent, $D_{\lambda} / L_{\lambda}$ is (divisible) abelian and $V_{\lambda} / D_{\lambda}$ is finite. The product $L$ of all the $L_{\lambda}$ is locally nilpotent and normal in $G$, and the product $D / L$ of all $D_{\lambda} L / L$ is likewise locally nilpotent and $G$-invariant. By two applications of Proposition 2, $D$ is soluble. Now $V / D$ is the product of $G$-invariant finite subgroups $V_{\lambda} D / D$, so $V / D$ is a periodic $F C$-group and its finite residual $R / D$ is therefore central.

Certainly $V / R$ is residually finite and locally finite, and if it is not soluble-by-finite then, for every normal subgroup $N / R$ of finite index in $V / R$, we have $N \notin \mathfrak{i} \subseteq$ and hence every subgroup of $V / N$ subnormal, which gives $V / N$ nilpotent. Thus $V / R$ is residually nilpotent and hence locally nilpotent, and Proposition 2 gives the contradiction that $V / R$ is soluble.

Thus $V / R$ is soluble-by-finite and hence $V$ is soluble-by-finite, so there is a soluble $G$-invariant subgroup $U$ of finite index in $V$. If $V \notin \mathfrak{N}$ C then all subgroups of $G / U$ are subnormal and $G / U$ is soluble [6]. Thus $G$ is soluble, as required.

Proposition 3. Let $G$ be a locally finite group in which every subgroup is either subnormal or nilpotent-by-Chernikov. Then $G$ is soluble-by-finite, and is either soluble or nilpotent-by-Chernikov.

Proof. If $G \in \mathfrak{N} \subseteq$ then $G$ is soluble-by-finite, so let us assume that $G \notin \mathfrak{N C}$ and proceed to show that $G$ is soluble-by-finite. Notice that this will imply that $G$ is soluble, for every finite image of $G$ has all subgroups subnormal and is therefore nilpotent (and hence soluble). Let $V$ be as in Lemma 4 and suppose for a contradiction that $G$ is not soluble-by-finite. Then $V \in \mathfrak{N} C$, and if $N / V$ is a nontrivial normal subgroup of $G / V$ then $N \notin \mathfrak{R}$, so every subgroup of $G / N$ is subnormal and $G / N$ is soluble and locally nilpotent. Let $M / V$ denote the intersection of all such normal subgroups $N / V$. Then $G / M$ is residually (locally nilpotent) and locally 
finite, so $G / M$ is locally nilpotent and hence soluble, by Proposition 2 . If $M=V$ then $G / V$ is soluble, while if $M \neq V$ then $M / V$ is the soluble residual of $G / V$ and Lemma 3 gives $G / V$ soluble-by-finite. In either case we have $G$ soluble-by-finite-by-soluble-by-finite and therefore soluble-byfinite.

Proof of Theorem 1. Assume the result false and let $R$ denote the soluble residual of $G$; by Lemma $3 G / R$ is not soluble. Now a soluble-byfinite group $F$ that is residually soluble is soluble, since $F$ modulo its soluble radical is finite and residually soluble and hence soluble. Thus $G / R$ is locally soluble, and there is no loss in supposing that $G$ is locally soluble.

Let $H_{1}$ denote the locally nilpotent radical of $G$ and, for each $i \geq 1$, let $H_{i+1} / H_{i}$ be the locally nilpotent radical of $G / H_{i}$; set $H=\cup_{i=1}^{\infty}\left(H_{i}\right)$. Now each $H_{i}$ is soluble, by Proposition 2 , and if $H_{i} \notin \mathfrak{N} \in$ for some $i$ then every subgroup of $G / H_{i}$ is subnormal and so $G / H_{i}$ is soluble, giving the contradiction that $G$ is soluble. Thus $H$ is contained in the subgroup $V$ of $G$ that is defined in Lemma 4 , and since $V \in \mathfrak{N} \in$ so is $H$. But then $H=H_{d}$ for some $d$; factoring, we may assume that $G$ has trivial locally nilpotent radical. Thus $G$ has no nontrivial subnormal soluble subgroups and hence no subnormal $\mathfrak{N}$ C -subgroups; so, among the nontrivial subgroups of $G$, the subnormal subgroups, the insoluble subgroups and the non- $\Re(5$ subgroups coincide. If $1 \neq N \triangleleft G$ then $G / N$ has all subgroups subnormal and is therefore soluble and locally nilpotent. Note that every finitely generated subgroup of $G$ is in $\mathfrak{N C}$ (and is therefore nilpotent-by-finite).

Now let $G_{0}$ be the intersection of all nontrivial normal subgroups $S$ of $G$ with $G / S$ torsion-free and locally nilpotent. Suppose that $G_{0}=1$, and let $X$ be an arbitrary finitely generated subgroup of $G$. There is a normal nilpotent subgroup $Y$ of $X$ of class $c$, say, such that $X / Y$ is finite. Every torsion-free nilpotent image of $X$ is nil-c and hence $X$ is nil-c. But then $G$ is locally nilpotent and Proposition 2 gives the contradiction that $G$ is soluble. It follows that $G_{0} \neq 1$ and hence that $G / G_{0}$ is locally nilpotent. If $N$ is an arbitrary nontrivial normal subgroup of $G_{0}$ then $G^{(d)} \leq N$ for some integer $d$, by Lemma 1 , and since $G / G^{(d)}$ is locally nilpotent we see that $G_{0} / G^{(d)}$ is the torsion subgroup of $G / G^{(d)}$, and so every proper image of $G_{0}$ is soluble, periodic and locally nilpotent. We may assume that $G=G_{0}$.

Next, let $G_{1}$ be the intersection of all nontrivial normal subgroups of $G$. Then $G_{1}$ is the soluble residual of $G$ and $G / G_{1}$ is insoluble, by Lemma 3 . It follows that $G_{1}=1$. 
By Proposition 1 there is an insoluble subgroup $K$ of $G$, a positive integer $r$ and a finitely generated subgroup $F$ of $K$ such that every insoluble subgroup of $K$ containing $F$ has defect at most $r$ in $K$. Let $N$ be an arbitrary nontrivial normal subgroup of $K$. Then $N$ is subnormal in $G$ and so $N \notin \Re\left(5\right.$ and, again by Lemma 1 , we have $G^{(d)} \leq N$ for some integer $d$, and $G / G^{(d)}$ is periodic and locally nilpotent, as therefore is $K / N$. Every subgroup of $K / N$ that contains $F N / N$ has defect at most $r$ in $K$, and we see too that $F N / N$ is finite. By Theorem 0.2 of [4], there is an integer $b$ depending only on $r$ such that $L:=\gamma_{b}(K)$ is finite modulo $N$. Similarly, if $M$ is an arbitrary nontrivial normal subgroup of $L$ then, since $K$ and hence $M$ is subnormal in $G$ there is a term $G^{(e)}$ of the derived series of $G$ contained in $M$, and since $F G^{(e)} / G^{(e)}$ is a finite subgroup of the locally nilpotent group $K / G^{(e)}$ it follows from [4] that $L / M$ is finite and nilpotent. In particular, $L / L^{\prime}$ is finite and so there is a finitely generated subgroup $U$ of $L$ such that $L=U L^{\prime}$. But then $L=U \gamma_{i}(L)$ for every positive integer $i$, and if $U$ has derived length at most $d$ then so has $L / \gamma_{i}(L)$ for every $i$. Since $G$ is residually soluble so is $L$, and as $L / M$ is finite nilpotent for every nontrivial normal subgroup $M$ of $L$ it follows that $L^{(d)}=1$ and $L$ is soluble. This gives the final contradiction that $G$ is soluble, and the proof of the theorem is complete.

Proof of Theorem 2. Supposing the result false, we may assume by Lemma 3 that $G$ is residually soluble, and hence that every $\mathfrak{N} \mathfrak{C}_{\text {-subgroup }}$ of $G$ is soluble. If $N$ is an arbitrary normal subgroup of $G$ with $G / N$ soluble then $N$ is not soluble and so it is not in $\mathfrak{N}$. But then every subgroup of $G / N$ is subnormal of defect at most $n$ and so $G / N$ is nilpotent of class depending only on $n$ [8]. Since the intersection of all such $N$ is trivial we have $G$ nilpotent and hence soluble, a contradiction.

\section{REFERENCES}

[1] A. O. AsAR, Locally nilpotent p-groups whose proper subgroups are hypercentral or nilpotent-by-Chernikov, J. London Math. Soc., 61 (2000), pp. 412422.

[2] C. J. B. BRookes, Groups with every subgroup subnormal, Bull. London Math. Soc., 15 (1983), pp. 235-238.

[3] C. CASOLO, On the structure of groups with all subgroups subnormal, J. Group Theory, 5 (2002), pp. 293-300.

[4] E. Deтомi, Groups with many subnormal subgroups, J. Algebra, 264 (2003), pp. 385-396. 
[5] W. MöHREs, Torsionsfreie Gruppen, deren Untergruppen alle subnomal sind, Math. Ann., 84 (1989), pp. 254-259.

[6] W. MöHREs, Auflösbarkeit von Gruppen, deren alle Untergruppen subnormal sind, Arch. Math., 54 (1990), pp. 232-235.

[7] F. NAPOLitani - E. Pegoraro, On groups with nilpotent-by-Černikov proper subgroups, Arch. Math., 69 (1997), pp. 89-94.

[8] J. E. Roseblade, On groups in which every subgroup is subnormal, J. Algebra, 2 (1965), pp. 402-412.

[9] H. SMITH, Torsion-free groups with all non-nilpotent subgroups subnormal, Topics in Infinite Groups, Quaderni di Matematica, 8 (2002), pp. 297-308.

[10] H. SмIтH, Groups with all non-nilpotent subgroups subnormal, Topics in Infinite Groups, Quaderni di Matematica, 8 (2002), pp. 309-326.

Manoscritto pervenuto in redazione il 26 maggio 2011. 